\title{
Case report: subcutaneous Exophiala xenobiotica infection successfully treated with a combined medical and surgical approach
}

\begin{abstract}
Exophiala xenobiotica is a black yeast from the Exophiala genus. Literature suggests it has consistently been underdiagnosed in recent years, because of poor microbiological identification techniques and the discovery of multiple new species within the genus. Exophiala xenobiotica most commonly causes subcutaneous infections. It often thrives in damp places, and places where there are aromatic compounds. This report describes a multi-comorbid patient including previous basal cell carcinoma and prostate cancer who developed this infection. An 88-year-old caucasian man presented to the orthopaedic clinic with a lump on the dorsum of his right wrist, he was given a provisional diagnosis of a ganglion cyst. The lesion had been there for two years and had steadily increased in size. The patient had no pain or systemic symptoms and was not prescribed any immunosuppressive medication. After the yeast was isolated, he was prescribed an eight-week course of itraconazole, with surgical excision of the lesion two weeks into the course. The procedure was a success, and the lesion showed no signs of recurrence at the six-week follow up. This case report adds to the literature supporting a combined surgical and medical approach for the treatment of subcutaneous Exophiala xenobiotica infections.
\end{abstract}

Keywords: Exophiala xenobiotica, treatment, combined approach
Volume 9 Issue 5 - 202I

\author{
Harjas Singh Shinmar,' Emily Macnaughton ${ }^{2}$ \\ 'Department of Surgery, Isle of Wight NHS Trust, United \\ Kingdom \\ ${ }^{2}$ Department of Microbiology, Isle of Wight NHS Trust, United \\ Kingdom
}

Correspondence: Harjas Singh Shinmar, Department of Surgery, Isle of Wight NHS Trust, United Kingdom,

Tel+447859997963, Email harjas.shinmar@nhs.net

Received: September 01, 2021 | Published: September 28, 2021

\section{Case presentation}

An 88-year-old gentleman was referred to the local orthopaedic team after complaining of a cystic growth the right dorsum of his wrist progressing over two years. He reported that before the lump presented, he had scratched his wrist whilst gardening. The patient reported no systemic symptoms.

\section{Past medical history}

His past medical history included hypertension, polymyalgia rheumatica, a malignant melanoma of his lower leg, a facial basal cell carcinoma, cerebellar haemorrhage, and carcinoma of the prostate and gallstones. He was not prescribed any immunosuppressant medications. He denied any alcohol intake and did not smoke.

\section{Patient examination}

On examination there was a cystic mass $(44 \mathrm{~mm} * 33 \mathrm{~mm} * 25 \mathrm{~mm})$ on the dorsum of the patient right wrist. It was fluctuant and swollen with no overlying erythema. The lump moved with the underlying tissues on finger extension. The patient had no constitutional symptoms of infection and local lymph nodes were not palpable.

\section{Differential diagnoses}

The key differential diagnoses for the wrist lesion included a sebaceous cyst, a ganglion cyst, lipoma, neoplasm or vascular malformation.

\section{Management plan}

An aspirate of the ganglion was sent for culture and sensitivity. Because of the patient's comorbidities it was initially thought that he would not be suitable for a general anaesthetic and conservative management was planned.

\section{Microbiology}

Local laboratory results: $5 \mathrm{mls}$ of frank pus, with +++ white cells and +++ yeast. The yeast was submitted to the PHE Mycology Reference Laboratory in Bristol for identification and susceptibility testing.

The following report came back:

Exophiala xenobiotica

Amphotericin B: $\mathrm{MIC}=0.5 \mathrm{mg} / \mathrm{l}$ sensitive

Itraconazole:

$\mathrm{MIC}=0.25 \mathrm{mg} / 1$

Voriconazole

$$
\mathrm{MIC}=4 \mathrm{mg} / \mathrm{l} \text { intermediate }
$$

$* \mathrm{MIC}=$ minimum inhibitory concentration ${ }^{1}$

\section{Further management}

Following these results, the patient was prescribed $200 \mathrm{mg}$ of itraconazole once a day for two weeks preceding an excisional biopsy of the lesion. The patient was then to have an excisional biopsy of the lesion. The patient then completed a further six weeks of itraconazole post operatively.

\section{Further results}

Repeat pus sample collected during the excision demonstrated a black fungal organism with budding yeast hyphae. Histological analysis of the lesion reported a nodule of pale fibrous tissue measuring $44 * 35 * 25 \mathrm{~mm}$, on sectioning this is a unilocular cyst 
with wall measuring up to $4 \mathrm{~mm}$ in thickness and the internal surface appears inflamed and ulcerated.

\section{Discussion}

Exophiala Xenobiotica is a black yeast from the genus Exophiala, that often grows in environments which are moist, hot with an abundance of toxic hydrocarbons. ${ }^{2}$ Exophiala xenobiotica comes from the Exophilia genus which has 16 different species identified within in it. ${ }^{3}$ The yeast is predominantly responsible for causing subcutaneous infections in humans ${ }^{2,4}$ but has also been shown to infect other sites including blood, dialysis fluid, intra-ocular tissues and mucous membranes. ${ }^{2}$ It has also been found to cause systemic infections fish. ${ }^{5}$ Although it commonly causes skin infections in humans, it is rarely responsible for systemic infections, like the other members of its genus. ${ }^{6}$

Two published case reports demonstrate infections in an HIV infected patient, ${ }^{2}$ and a patient with non-Hodgkin's lymphoma, ${ }^{6}$ however literature suggests it is more commonly associated with healthy individuals and innately immunocompromised patients, rather than those with acquired immunodeficiencies. ${ }^{2}$

In a study by Zeng and colleagues in $2009,{ }^{3}$ where over 188 samples of Exophilia infections were analysed, Exophiala xenobiotica was responsible for $20 \%$ of infections in this study population. Although the patient in question did not currently have any immunosuppressive therapy prescribed at the time of infection, his multi-comorbid status will have weakened his immune system, which may have made him more susceptible to develop this infection.

Current literature suggests that Amphotericin B, voriconazole, itraconazole, posocanazole is effective treatments for Exophiala xenobiotica infection (ibid), with the azole antifungals being the most effective (ibid). In addition to this a combined medical and surgical approach is recommended for complete eradication of the yeast. ${ }^{3,6}$

\section{Conclusion}

Aspiration of the cyst showed that the fungus Exophiala xenobiotica was responsible for the current presentation. An eightweek course of Itraconazole pre and post operatively was given to the patient. When the patient was followed up in the orthopaedic clinic his wound had healed nicely and there was no further recurrence of swelling or infection. The patient however died 6 months after his procedure likely due to an unrelated intrabdominal infection and progressive metastatic prostate cancer. This case report adds to the literature supporting a combined surgical and medical approach towards the treatment of Exophiala xenobiotica cutaneous infections.

\section{Scope for further research}

As Morio and colleaugues in $2012^{2}$ conclude, there is no consensus on what the treatment for Exophilia Xenobiotica infections should be. This can be supported by further case reports, building up the evidence base to analyse treatment strategies for this infection including cases where longer term follow up is possible.

\section{Acknowledgments}

None.

\section{Conflicts of interest}

Authors declare that there is no conflict of interest.

\section{References}

1. Andrews JM. Determination of minimum inhibitory concentrations. Journal of Antimicrobial Chemotherapy. 2001;48(Suppl 1):5-16.

2. Morio F, Le Berre J, Garcia-Hermoso D, et al. Phaeohyphomycosis due to Exophiala xenobiotica as a cause of fungal arthritis in an HIV - infected patient. Medical Mycology. 2012;50(5):513-517.

3. Zeng JS, Sutton DA, Fothergill AW, et al. Spectrum of Clinically Relevant Exophiala Species in the United States. Journal of Clinical Microbiology. 2007;45(11):3713-3720.

4. De Hoog GS, Zeng M, Harrak MJ, et al. Exophiala xenobiotica sp. Nov., an opportunistic black yeast inhabiting environments rich in hydrocarbons. Antonie van Leeuwenhoek. 2006;90(3):257-268.

5. Munchan C, Kurata O, Wada S, et al. Exophiala Xenobiotica infection in cultured striped jack, Pseudocaranx dentex (Bloch \& Schneider, in Japan. Journal of Fish Diseases. 2009;32(10):893-900.

6. Aoyama Y, Nomura M, Yamanaka S, et al. Subcutaneous phaeohyphomycosis caused by Exophilia xenobiotica in a non- Hodgkin lymphoma patient. Medical Mycology. 2009;47(1):95-99. 